\title{
基于等承载思想的低匹配 $\mathbf{T}$ 型接头设计 ${ }^{*}$
}

\author{
郭军礼董志波 刘雪松王苹方洪渊 \\ (哈尔滨工业大学先进焊接与连接国家重点实验室＼cjkstart哈尔滨１50001)
}

\begin{abstract}
摘要: 针对低匹配焊接接头承载能力不足的问题, 基于等承载思想建立等承载基本理论, 通过定义广义匹配比和广义力学集 中系数, 提出广义力学集中系数与广义匹配比相等为等承载的实现条件。构建等承载设计的一般方法: 明确接头失效形式, 确定目标失效模式; 确定接头危险位置; 构建承载能力与几何参数、材料力学性能关系式; 分别求解焊缝临界曲线并取包络 线。以承受静拉伸的 $\mathrm{T}$ 型接头为例验证了以上方法的可行性: 失效形式为静载断裂, 按照全面屈服(焊缝)断裂模式进行设计, 给出明确的实现条件; 建立理论应力集中系数与几何参数的关系式; 结合 $\mathrm{T}$ 型接头几何特点给出求解步骤; 建立等承载 $\mathrm{T}$ 型 接头弹塑性有限元模型, 接头的断裂模式为全面屈服(焊缝)断裂, 承载能力达到了母材的屈服水平。结果表明等承载基本理 论和方法具有一定的普适性, 能为更多的焊接接头等承载设计问题提供解决方案。
\end{abstract}

关键词: 低匹配; 等承载; 理论; 设计方法; $\mathrm{T}$ 型接头

中图分类号: TG156

\section{Design of Undermatching $T$ Joints Based on the Thought of Equal Load Carrying Capacity}

\author{
GUO Junli DONG Zhibo LIU Xuesong WANG Ping FANG Hongyuan
}

(State Key Laboratory of Advanced Welding and Joining, Harbin Institute of Technology, Harbin150001)

\begin{abstract}
To improve the load-carrying capacity of undermatching joints, the basic theory of equal load-carrying capacity (ELCC)design is established based on the thought of ELCC. The concepts of generalized mechanics concentration factor(GMCF) and generalized mis-match ratio (GMMR)are defined, so the realization condition of ELCC is presented, i.e.make GMC Fequal to GMMR. The general approach of ELCC design is proposed: (1) ascertain the failure form of joints, determine the target failure mode; (2) determine the failure position; (3) obtainthe relational expression betweenload carrying capacity and geometrical parameters, mechanical properties of materials; (4) obtain the critical curve of weld seam respectively, then take the envelope curve as the final solution. According to the procedure proposed, the ELCCTjoints with tensile load are designed. The failure form is static load fracture, the target failure mode isoverall yield (weld seam) fracture mode, thenthe realization condition of ELCC is specialized. Calculate the theoretical stress concentration factorof different geometric parameters through numerical method,establish the equation of them, then put forward the equal load-carrying capacity design strategy for T-joint joints. An elastic-plastic finite element model of one ELCC T-joint is built, and results show that the joint fractures in overall yield (weld seam) fracture model, its load-carrying capacity reachesthe yield strength of base metal.The results indicate that the theory and approachproposedarefeasible and can provide solutions to more ELCC problems.
\end{abstract}

Key words: undermatching; equal load carrying; theory; design method; $\mathrm{T}$ joints

\section{0 前言}

提高焊接结构可靠性和减轻焊接结构重量的需 求越来越迫切, 因此高强钢的应用日益广泛。但是 高强钢焊接时存在冷裂纹(氢致裂纹)、热影响区的 脆化和软化、接头疲劳强度不足等主要问题 ${ }^{[1-3]}$, 严

* 国家自然科学基金资助项目(51875131)。20180402 收到初稿, 20180718 收到修改稿
重限制了高强钢效能的发挥。其中冷裂纹问题尤为 显著, 是高强钢焊接接头早期失效的主要原因之一。 目前防止冷裂纹的措施 ${ }^{[4-5]}$ 主要有: (1) 开发专用焊 材, 如低氢或超低氢焊接材料, 或使用低碳马氏体 和奥氏体-马氏体双相型抗裂焊材焊接高强钢得到 的接头性能较好, 但是专用焊材的开发周期较长, 成本较高; (2) 进行焊前预热和焊后消氢处理, 虽然 可以控制冷裂纹，但同时降低了焊接热影响区强韧 性, 加剧其软化和脆化现象 ${ }^{[6-8]}$ 。此外, 杨建国研究 
了 $30 \mathrm{CrMnSi}$ 钢焊接冷裂纹形成的机制, 提出控制 热裂纹来防止冷裂纹的有益思路, 其控制工艺有待 进一步研究 ${ }^{[9]}$ 。采用强度低于母材、塑韧性高的焊 材焊接高强钢, 更容易获得高质量的接头, 冷裂纹 得到控制, 接头塑韧性显著提高 ${ }^{[10-12]}$ 。低匹配接头 可视为 “软夹层” 接头, 在拘束强化、冶金强化作 用下 ${ }^{[5,13]}$, 接头的强度高于焊缝金属, 低于母材。 当夹层厚度足够小时, 接头强度甚至可达到 “实际 等强”, 但塑性显著下降。对于大多数低匹配接头, 其承载能力还是存在明显不足, 仍然是焊接结构的 薄弱环节。焊接接头(尤其是低匹配接头)的设计标 准尚不完善, 设计人员对焊接结构的生产实际和接 头的承载特点、失效模式缺乏准确的认识, 都造成 焊接结构的可靠性降低和人力物力的浪费。由于传 统的设计理念和设计方法无法解决高强钢焊接产品 设计和生产中出现的新问题 ${ }^{[14-15]}$, 有研究者 ${ }^{[16-18]}$ 打 破 “焊接接头的承载能力低于母材” 这一思维定势, 提出了等承载焊接接头设计的思想, 通过调整接头 的几何参数, 使低匹配焊接接头与母材具有相同的 承受静载荷、动载荷、交变载荷等的能力, 具有与 母材相同的抵抗变形、断裂等的能力。赵智力等 ${ }^{[18-20]}$ 以静载断裂为失效形式, 建立了对接接头静拉伸等 承载理论和实现条件, 借助数值方法建立等承载实 现条件与接头几何参数的关系, 给出了对接接头静 拉伸等承载设计准则。程荣龙 ${ }^{[21]}$ 对承受静拉伸的搭 接接头进行了等承载设计研究, 建立了相应设计准 则。王佳杰等 ${ }^{[22-23]}$ 分别以塑性变形失效、静载断裂 为失效形式, 建立了对接接头静弯曲等承载理论和 实现条件, 采用解析法建立等承载实现条件与接头 几何参数的关系, 给出了对接接头静弯曲等承载设 计准则。王涛 ${ }^{[24}$ 以静载断裂为失效形式, 从断裂力 学应力强度因子角度建立了带裂纹对接接头静拉伸 等承载实现条件, 综合运用解析法和数值法建立了 应力强度因子与接头几何参数的关系, 最终给出了 相应的等承载设计准则。雷靖 ${ }^{[25]}$ 用数值法计算了不 同形状参数对接接头的动态应力强度因子, 实现了 接头抗冲击等承载能力。基于等承载的基本思想, 总结已有等承载研究的共性规律, 本文旨在给出等 承载实现条件的普遍形式, 以便将等承载设计运用 于其它接头形式、载荷类型、失效形式、应用场合; 并给出焊接接头等承载设计研究的一般方法。

\section{1 等承载基本思想和理论}

承载能力是指整个接头所能承受的载荷, 与材 料的强度、接头几何形状、载荷形式等都有关系。
提高焊接接头的承载能力, 可以通过提高材料的强 度实现, 也可以通过调整接头的几何参数(增大承载 面积、缓和应力集中)实现。

如果能使焊接接头的承载能力与平滑母材的承 载能力相同, 则在焊接结构的设计中, 可以不必考 虑因焊接接头的存在而额外增加母材的厚度, 并且 提高了结构的可靠性。鉴于此，低匹配焊接接头等 承载能力的设计思想为

$$
P^{\mathrm{J}}=P^{\mathrm{B}}
$$

式中 $P^{\mathrm{J}}$ 一焊接接头失效时所能承受的载荷;

$P^{\mathrm{B}}$ ——平滑母材失效时所能承受的载荷。

也就是要求焊缝的承载能力不低于平滑母材

$$
P^{\mathrm{W}} \geqslant P^{\mathrm{B}}
$$

式中 $P^{\mathrm{W}}$ 一一接头失效时焊缝所能承受的载荷。

$P^{\mathrm{B}} 、 P^{\mathrm{W}}$ 可由母材、焊缝金属几何参数和材料力 学性能的函数分别表达, 即

$$
\begin{aligned}
& P^{\mathrm{B}}=F_{\mathrm{G}^{* \mathrm{M}}}^{\mathrm{B}} \\
& P^{\mathrm{W}}=F_{\mathrm{G}^{* \mathrm{M}}}^{\mathrm{W}}
\end{aligned}
$$

式中 $F_{\mathrm{G}^{*} \mathrm{M}}^{\mathrm{B}}$ - 一母材几何参数和材料力学性能的 函数;

$F_{\mathrm{G}^{*} \mathrm{M}}^{\mathrm{W}}$ 一一焊缝几何参数和材料力学性能的 函数。

将式(3)、(4)代入式(2)有

$$
F_{\mathrm{G}^{*} \mathrm{M}}^{\mathrm{B}} \leqslant F_{\mathrm{G}^{*} \mathrm{M}}^{\mathrm{W}}
$$

式(5)说明, 当材料参数确定, 等承载能否实现 仅取决于接头的几何参数。式(5)即等承载实现条件 的一般形式, 将母材、焊缝材料力学性能和母材尺 寸代入, 求解可得等承载接头焊缝的几何参数。

进一步, 如果对于焊接接头的母材和焊缝金属 分别有

$$
\begin{aligned}
P^{\mathrm{B}} & =F_{\mathrm{G}}^{\mathrm{B}} \times F_{\mathrm{M}}^{\mathrm{B}} \\
P^{\mathrm{W}} & =F_{\mathrm{G}}^{\mathrm{W}} \times F_{\mathrm{M}}^{\mathrm{W}}
\end{aligned}
$$

式中 $F_{\mathrm{G}}^{\mathrm{B}}, F_{\mathrm{G}}^{\mathrm{W}}$ 一一母材、焊缝金属几何参数的 函数;

$F_{\mathrm{M}}^{\mathrm{B}}, F_{\mathrm{M}}^{\mathrm{W}}$ —母材、焊缝材料力学性能的 函数。

将式(6)、(7)代入式(2)并整理有

$$
\frac{F_{\mathrm{G}}^{\mathrm{B}}}{F_{\mathrm{G}}^{\mathrm{W}}} \leqslant \frac{F_{\mathrm{M}}^{\mathrm{W}}}{F_{\mathrm{M}}^{\mathrm{B}}}
$$

式(8)的右边仅与材料力学性能有关, 定义其为 广义匹配比 $\mu_{\mathrm{N}}$

$$
\mu_{\mathrm{N}}=\frac{F_{\mathrm{M}}^{\mathrm{W}}}{F_{\mathrm{M}}^{\mathrm{B}}}
$$


左边仅与接头的几何参数有关, 定义其为广义 力学集中系数 $k_{\mathrm{N}}$

$$
k_{\mathrm{N}}=\frac{F_{\mathrm{G}}^{\mathrm{B}}}{F_{\mathrm{G}}^{\mathrm{W}}}
$$

那么等承载的实现条件可简化为

$$
k_{\mathrm{N}} \leqslant \mu_{\mathrm{N}}
$$

式(11)表明，等承载能否实现取决于接头的广 义力学集中系数和广义匹配比。广义力学集中系数 可直接用来进行等承载设计, 可大大简化设计的过 程。此时等承载接头的几何参数仅取决于广义匹配 比的取值, 将广义匹配比、母材尺寸代入式(11), 求解可得等承载接头焊缝的几何参数。

在文献 $[18,21]$ 研究的情形中, $\mu_{\mathrm{N}}$ 表现为屈服 强度匹配比, 相应的 $k_{\mathrm{N}}$ 表现为理论应力集中系数; 在文献[22]研究的情形中, $\mu_{\mathrm{N}}$ 表现为当量应力比(即 当量强度匹配比), 相应的 $k_{\mathrm{N}}$ 表现为应力集中系数; 在文献[24-25]研究的情形中, $\mu_{\mathrm{N}}$ 表现为临界(动态) 应力强度因子匹配比, 相应的 $k_{\mathrm{N}}$ 表现为(动态)应力 强度因子集中系数。可见, 在不同的情形下, $\mu_{\mathrm{N}}$ 和 $k_{\mathrm{N}}$ 密切相关, 且均有不同的形式, 其具体形式由接 头设计的目标失效模式决定。

\section{2 焊接接头等承载设计的一般方法}

理论上, 对于任意情形, 当式(5)或式(11)中等 号成立时存在唯一的焊缝临界曲线满足等承载要 求。但是, 由于焊接接头失效形式和承受载荷的复 杂性、不确定性, 受限于当前研究水平, 复杂情形 下求解这一临界曲线非常困难, 需要采取措施进行 简化, 降低设计难度, 即等承载设计的一般方法。

\section{1 明确接头失效形式, 确定目标失效模式}

明确接头失效形式, 研究接头承载能力与失效 模式的关系, 进而确定目标失效模式。

焊接结构的失效形式可分为断裂失效、变形失 效、表面损伤失效三类。其中断裂失效包括静载断 裂、疲劳断裂、冲击断裂、蠕变断裂、应力腐蚀断 裂失效等; 变形失效包括弹性变形失效和塑性变形 失效; 表面损伤失效包括磨损、接触腐蚀、接触疲 劳等。根据结构应用的场合、重要的程度、要实现 的功能、承受的载荷类型等, 明确接头的失效形式。 同一种结构可同时有几种不同的失效形式, 在设计 接头时必须全部予以考虑。

接头的失效模式是指在确定的失效形式下焊缝 金属与母材力学响应阶段的组合。不同的失效模 式下, 接头的承载能力不同, 可作为等承载设计
的判据。

以静载断裂失效为例, 对焊接接头的失效模式 进行分析。

如图 1 所示接头中, $\varepsilon^{\prime}$ 为危险点的应变(即危险 截面上的应变的最高值), $\varepsilon^{\prime \prime}$ 为危险截面上的应变的 最低值, 远端母材的应变 $\varepsilon, \varepsilon_{\mathrm{s}}^{\mathrm{B}} 、 \varepsilon_{\mathrm{s}}^{\mathrm{W}}$ 分别为母材、 焊缝金属的屈服时的应变, 根据断裂时各参数的关 系, 接头的断裂模式可分为以下类别。

(焊缝)线弹性断裂

$$
\varepsilon_{\mathrm{s}}^{\mathrm{W}}>\varepsilon^{\prime}>\varepsilon^{\prime \prime}, \quad \varepsilon_{\mathrm{s}}^{\mathrm{B}}>\varepsilon
$$

(焊缝)弹塑性断裂

$$
\varepsilon^{\prime}>\varepsilon_{\mathrm{s}}^{\mathrm{W}}>\varepsilon^{\prime \prime}, \quad \varepsilon_{\mathrm{s}}^{\mathrm{B}}>\varepsilon
$$

焊缝屈服断裂

$$
\varepsilon^{\prime}>\varepsilon^{\prime \prime}>\varepsilon_{\mathrm{s}}^{\mathrm{W}}, \quad \varepsilon_{\mathrm{s}}^{\mathrm{B}}>\varepsilon
$$

全面屈服(焊缝)断裂

$$
\varepsilon^{\prime}>\varepsilon^{\prime \prime}>\varepsilon_{\mathrm{s}}^{\mathrm{W}}, \varepsilon>\varepsilon_{\mathrm{s}}^{\mathrm{B}}
$$

全面屈服断裂

$$
\varepsilon^{\prime}>\varepsilon^{\prime \prime}>\varepsilon_{\mathrm{s}}^{\mathrm{W}}, \varepsilon>\varepsilon_{\mathrm{s}}^{\mathrm{B}}
$$

全面屈服(母材)断裂

$$
\varepsilon^{\prime}>\varepsilon^{\prime \prime}>\varepsilon_{\mathrm{s}}^{\mathrm{W}}, \quad \varepsilon>\varepsilon_{\mathrm{s}}^{\mathrm{B}}
$$

(焊缝)弹塑性(母材)屈服断裂

$$
\varepsilon^{\prime}>\varepsilon_{\mathrm{s}}^{\mathrm{W}}>\varepsilon^{\prime \prime}, \quad \varepsilon>\varepsilon_{\mathrm{s}}^{\mathrm{B}}
$$

(焊缝)线弹性(母材)屈服断裂

$$
\varepsilon_{\mathrm{s}}^{\mathrm{W}}>\varepsilon^{\prime}>\varepsilon^{\prime \prime}, \quad \varepsilon>\varepsilon_{\mathrm{s}}^{\mathrm{B}}
$$

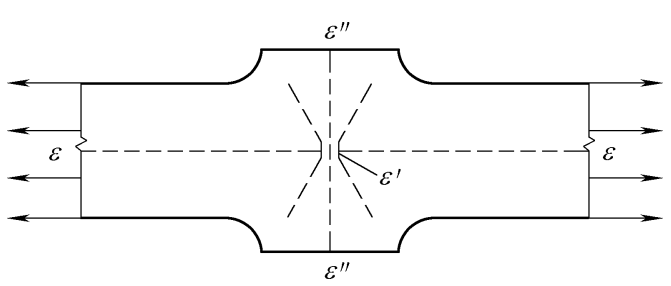

图 1 焊接接头拉伸试样

对于前两种断裂模式，断裂时应力很低，塑性 变形很小, 属于脆性断裂, 是不安全的断裂模式。 在焊缝屈服断裂模式下，结构虽然发生了一定的塑 性变形, 但主要集中在焊缝附近, 试件的大部分区 域尚未屈服, 母材的承载能力没有完全发挥, 属于 低应力破坏, 也是不安全的断裂模式。在全面屈服 (焊缝)断裂模式下, 试件整体产生塑性变形, 变形 较大, 母材的承载能力充分发挥, 达到了屈服强度, 属于延性断裂, 已是比较安全的断裂模式, 但是断 裂仍旧发生在焊缝, 接头的安全裕度比平滑母材要 小很多。在全面屈服断裂模式下, 焊缝和母材均完 
全屈服, 同时断裂, 母材的承载能力完全发挥, 达 到了抗拉强度, 且接头的安全裕度与平滑母材相等。 在全面屈服(母材)断裂模式下, 断裂发生在母材, 接头的安全裕度大于平滑母材, 即焊缝金属几余。 在后两种断裂模式下亦是如此, 只是焊缝金属更加 圥余。

接头的设计要确保安全性, 兼顾经济性, 所以 设计接头时的目标断裂模式最好为全面屈服断裂模 式, 设计出的等承载接头的断裂模式至少为全面屈 服断裂模式。

\section{2 确定接头危险位置}

即使在确定的载荷类型和失效形式下, 因载荷 不同, 结构的危险位置可能不同。例如, 同样是静 载断裂, 带余高的对接接头承受拉伸载荷时首先断 裂在焊根处(暂不考虑焊趾处), 承受弯曲载荷时首 先断裂在焊缝表面。

将同类型的载荷进一步细分为不同的单纯载 荷, 找到危险位置。显然, 结构可同时有多个危险 位置, 在设计接头时必须全部予以考虑。

\section{3 构建承载能力表达式}

每一确定的失效形式下, 母材、焊㖓承受某一 单纯载荷的能力可用几何参数、材料力学性能来表 征。构建承载能力与几何参数、材料力学性能关系 式, 即式(3)、(4)或式(6)、(7), 进而可得到等承载 实现条件式(5)或式(11)。

构建此类关系式的方法有解析法、数值法和经 验法。解析法推导严密, 关系式物理意义明确, 可 不提前限定焊缝形状, 是最可能求得焊缝临界曲线 的方法, 但是限于当前的研究水平, 解析法只能用 于几种简单的情形。数值法通过建立数值模型计算 得到大量数据, 拟合得到所需关系式, 过程简单, 可操作性强, 可同时对焊趾加以考虑, 但是建立数 值模型时需要限定焊缝形状。对于复杂的情形, 只 能采用经验法, 根据生产实践中积累的大量数据建 立经验公式。

\section{4 求解临界曲线}

分别解出所有的焊缝临界曲线, 取这些曲线的 包络线作为接头等承载设计焊缝的临界曲线。

如图 2 所示, 对情形 1, 焊缝的临界曲线为曲

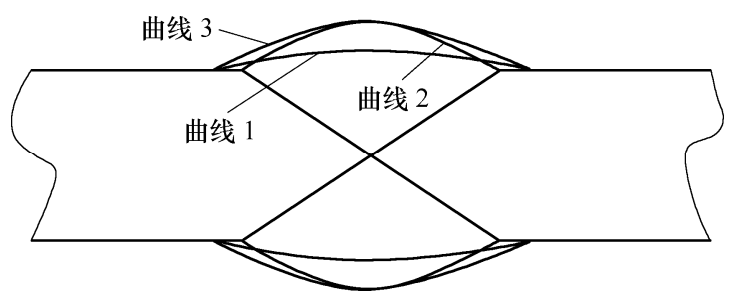

图 2 等承载设计焊缝临界曲线确定方法
线 1 , 对情形 2 , 焊缝的临界曲线为曲线 2 , 那么等 承载设计焊缝的临界曲线为曲线 1 和曲线 2 的包络 线即曲线 3 。

\section{3 焊接接头等承载设计应用实例}

承受静拉伸的 $\mathrm{T}$ 型接头形状如图 3 所示, 可以 确定失效形式为静载断裂。

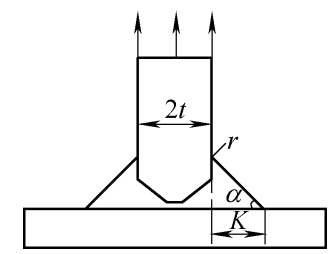

图 $3 \mathrm{~T}$ 型接头的形状方案

根据 $\mathrm{T}$ 型焊接接头的几何特点, 可实现最大焊 趾过渡圆弧半径受到焊趾角度和焊脚尺寸的限制, 关系式如下

$$
r_{\max } \leqslant \frac{K \tan \alpha}{\cos \alpha}
$$

如果按照全面屈服断裂模式进行设计, 则要求 焊趾处没有应力集中, 使得焊缝过大，导致其他问 题, 所以应按照全面屈服(焊缝)断裂模式进行设计, 使接头的承载能力达到母材屈服水平。

此时等承载实现条件如下。

按最大主应力计算

$$
\begin{gathered}
K_{\mathrm{t}}^{\mathrm{toe}} \leqslant K_{\mathrm{tc}}^{\mathrm{toe}}=\sqrt{\frac{\sigma_{\mathrm{b}}^{\mathrm{W}} \varepsilon_{\mathrm{b}}^{\mathrm{W}}}{\sigma_{\mathrm{b}}^{\mathrm{B}} \varepsilon_{\mathrm{b}}^{\mathrm{B}}}} \\
K_{\mathrm{t}}^{\text {root }} \leqslant K_{\mathrm{tc}}^{\text {root }}=\frac{\sigma_{\mathrm{b}}^{\mathrm{W}}}{\sigma_{\mathrm{s}}^{\mathrm{B}}}
\end{gathered}
$$

式中 $K_{\mathrm{t}}^{\mathrm{toe}}, K_{\mathrm{t}}^{\mathrm{root}}$ 一焊趾、焊根处的理论应力集 中系数;

$K_{\mathrm{tc}}^{\mathrm{toe}}, K_{\mathrm{tc}}^{\mathrm{root}}$ 一一焊趾、焊根处的临界理论应 力集中系数。

按 Von Mises 应力计算

$$
K_{\mathrm{t}}^{\mathrm{root}} \leqslant K_{\mathrm{tc}}^{\mathrm{root}}=\frac{\sigma_{\mathrm{s}}^{\mathrm{W}}}{\sigma_{\mathrm{s}}^{\mathrm{B}}}
$$

同时, 满足焊根处等承载设计实现条件的前提 下，焊缝金属的熔敷量越少越好，即求式(24)极小值

$$
A=\frac{1}{2} K^{2} \tan \alpha
$$

用数值法构建焊趾、焊根处理论应力集中系数 与几何参数关系式，进而进行等承载设计。

由对称性, 可取接头的 $1 / 2$ 进行计算, 划分平 面网格, 接头部分网格较细, 单元平均尺寸设为 
$0.125 \mathrm{~mm}$, 几何特性为平面应变类型, 单元类型四 节点平面应变单元, 进行线弹性分析。计算结果示 意见图 4。

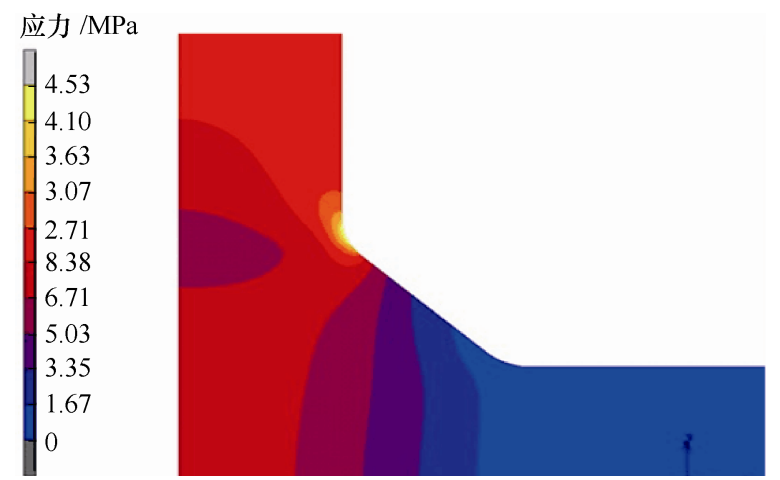

图 $4 \mathrm{~T}$ 型接头等效 Von Mises 应力分布示意图

图 5a、 $5 \mathrm{~b}$ 分别为焊脚尺寸取 $5 \mathrm{~mm} 、 7 \mathrm{~mm}$ 时按 等效 Von Mises 应力计算的焊趾、焊根处的理论应 力集中系数与焊趾过渡圆弧半径的关系曲线。图 $6 \mathrm{a} 、 6 \mathrm{~b}$ 分别为焊脚尺寸取 $5 \mathrm{~mm} 、 7 \mathrm{~mm}$ 时按最大主 应力计算的焊趾、焊根处的理论应力集中系数与焊 趾过渡圆弧半径的关系曲线。

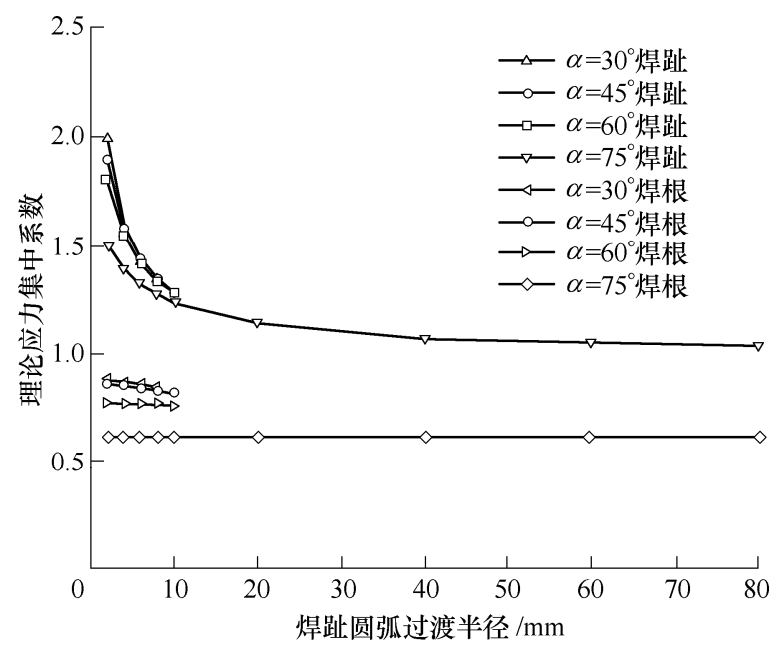

(a) $K=5 \mathrm{~mm}$

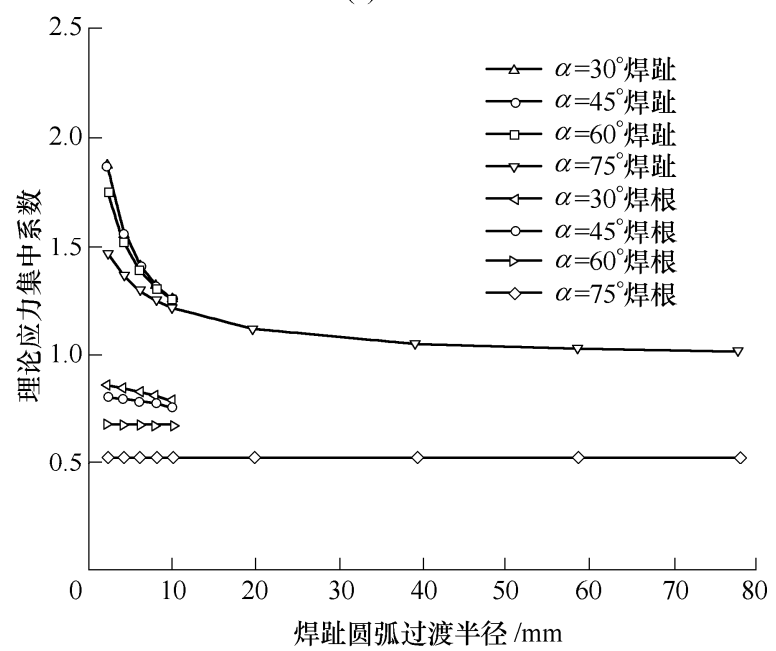

(b) $K=7 \mathrm{~mm}$

图 5 按等效 Von Mises 应力计算焊趾过渡圆弧半径的影响

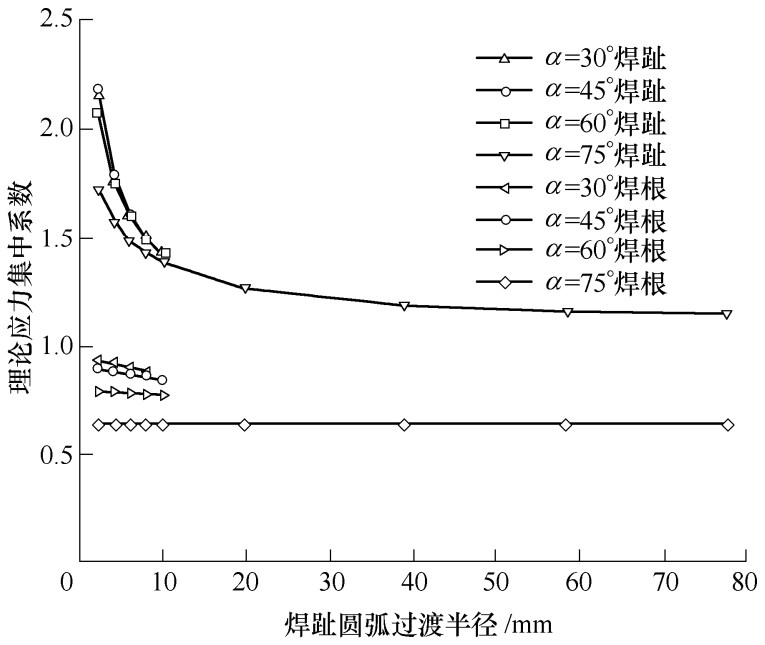

(a) $K=5 \mathrm{~mm}$

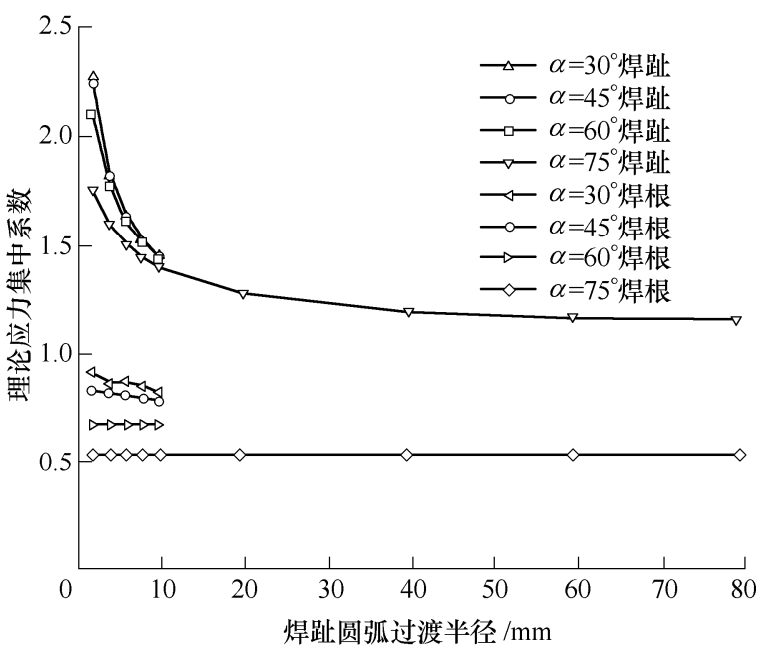

(b) $K=7 \mathrm{~mm}$

图 6 按最大主应力计算时焊趾过渡圆弧半径的影响

由图 5 可知, 当焊趾过渡圆弧半径较小时, 焊趾处的理论应力集中系数很大，随着焊趾过渡 圆弧半径的增大而显著下降，当焊趾过渡圆弧半 径较大时, 尽管焊脚尺寸和焊趾角度取值不同, 焊趾处的理论应力集中系数差别不大，趋于一致， 都接近于 1 , 随着焊趾过渡圆弧半径的增大变化很 小。焊根的应力集中系数随着焊趾过渡圆弧半径 的增大而线性下降, 且斜率随角度增大而增大, 最后接近于 0 。

图 7a、7b 分别为焊趾圆弧过渡半径取 $2 \mathrm{~mm} 、 8$ $\mathrm{mm}$ 时按等效 Von Mises 应力计算的焊趾、焊根处的 理论应力集中系数与焊趾角度的关系曲线。图 $8 \mathrm{a}$ 、 $8 \mathrm{~b}$ 分别为焊趾圆弧过渡半径取 $2 \mathrm{~mm} 、 8 \mathrm{~mm}$ 时按最 大主应力计算的焊趾、焊根处的理论应力集中系数 与焊趾角度的关系曲线。

由图 7、8 可知, 当焊趾过渡圆弧半径较小时, 理论应力集中系数随焊趾倾角角度的增加而减小, 呈指数衰减趋势。当焊趾过渡圆弧半径较大时, 焊 趾倾角对焊趾理论应力集中系数影响很小。 


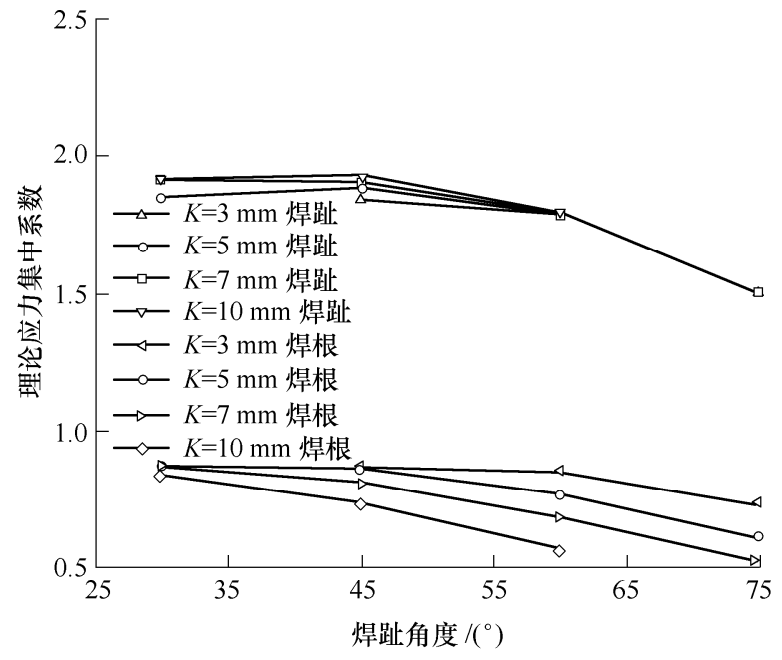

(a) $r=2 \mathrm{~mm}$

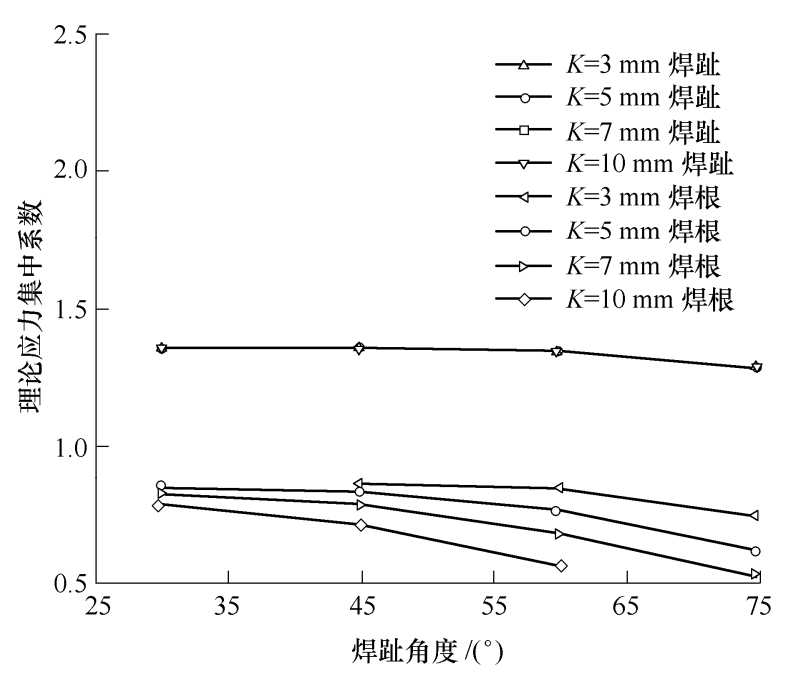

(b) $r=8 \mathrm{~mm}$

图 7 按等效 Von Mises 应力计算时焊趾角度的影响

图 9a、9b 分别为焊趾圆弧过渡半径取 $2 \mathrm{~mm}$ 、 $8 \mathrm{~mm}$ 时按等效 Von Mises 应力计算的焊趾、焊根处 的理论应力集中系数与焊接尺寸的关系曲线。图

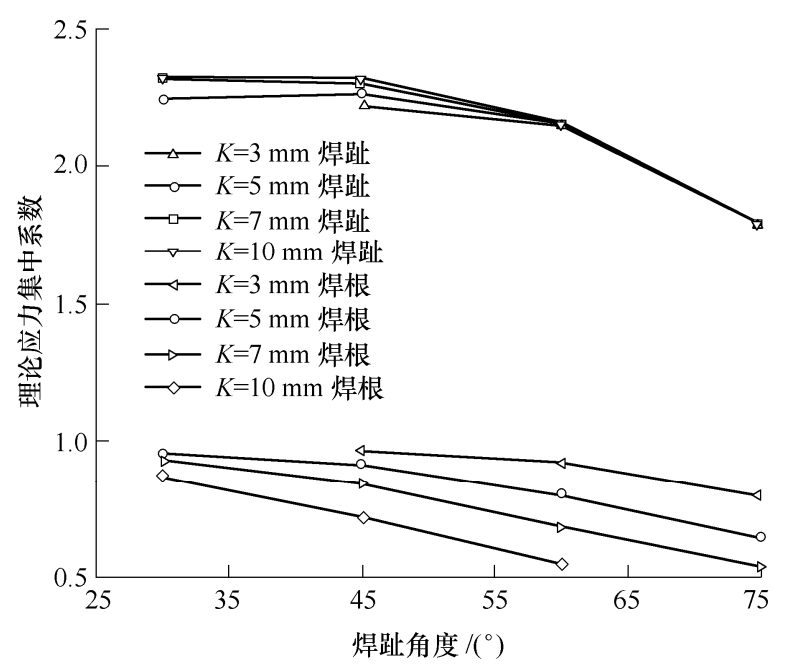

(a) $r=2 \mathrm{~mm}$

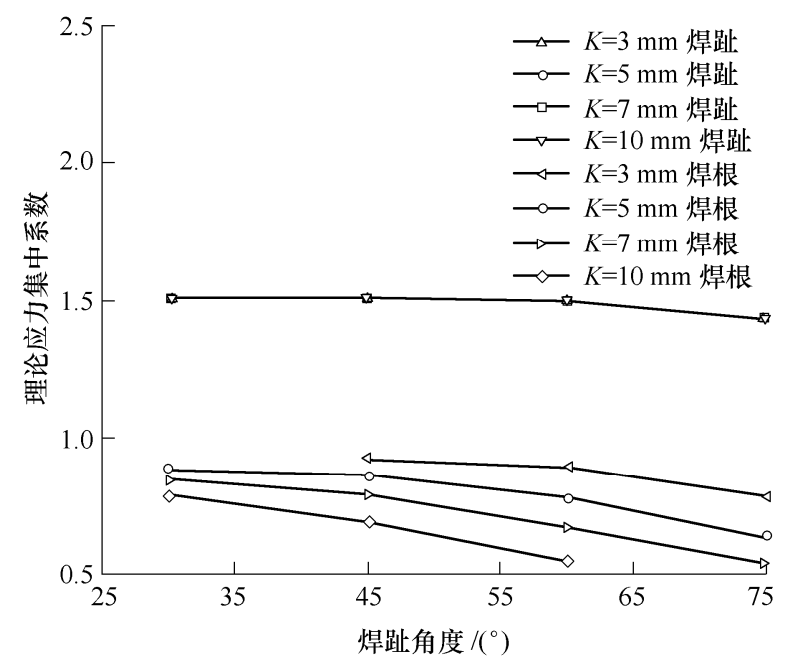

(b) $r=8 \mathrm{~mm}$

图 8 按最大主应力计算时焊趾角度的影响

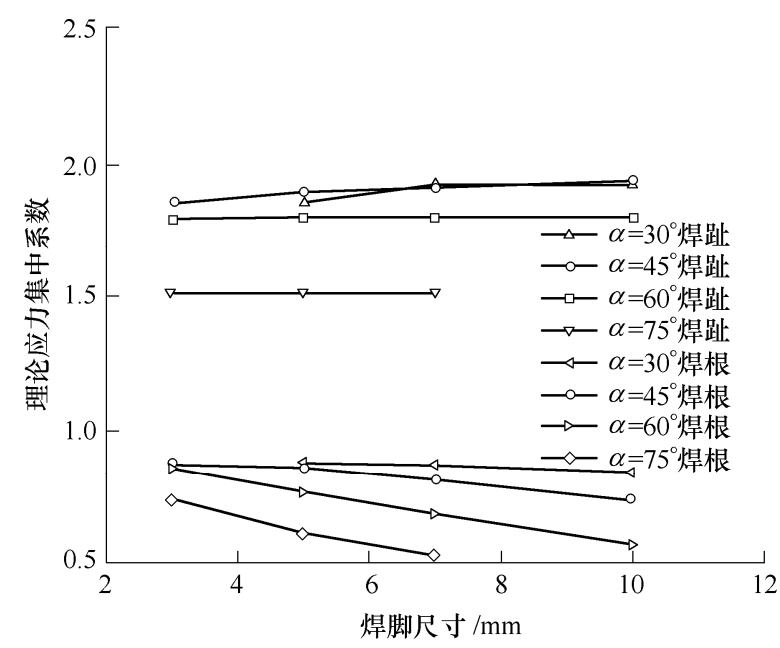

(a) $r=2 \mathrm{~mm}$

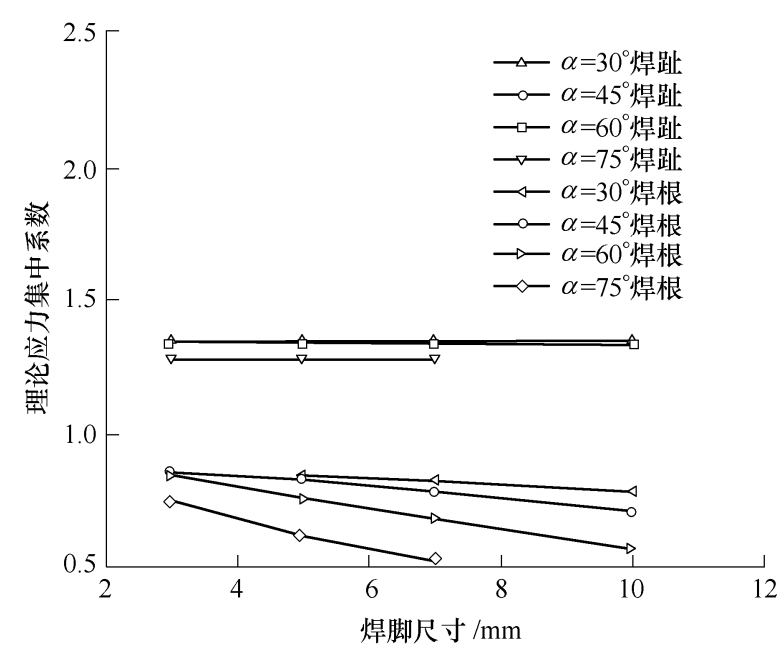

(b) $r=8 \mathrm{~mm}$

图 9 按等效 Von Mises 应力计算时焊脚尺寸的影响

$10 \mathrm{a} 、 10 \mathrm{~b}$ 分别为焊趾圆弧过渡半径取 $2 \mathrm{~mm} 、 8 \mathrm{~mm}$ 时按最大主应力计算的焊趾、焊根处的理论应力集 中系数与焊脚尺寸的关系曲线。 


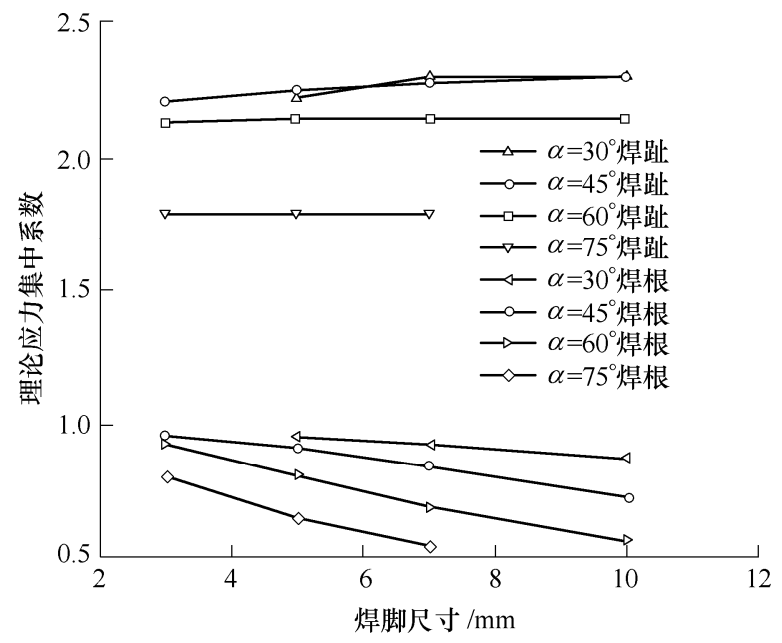

(a) $r=2 \mathrm{~mm}$

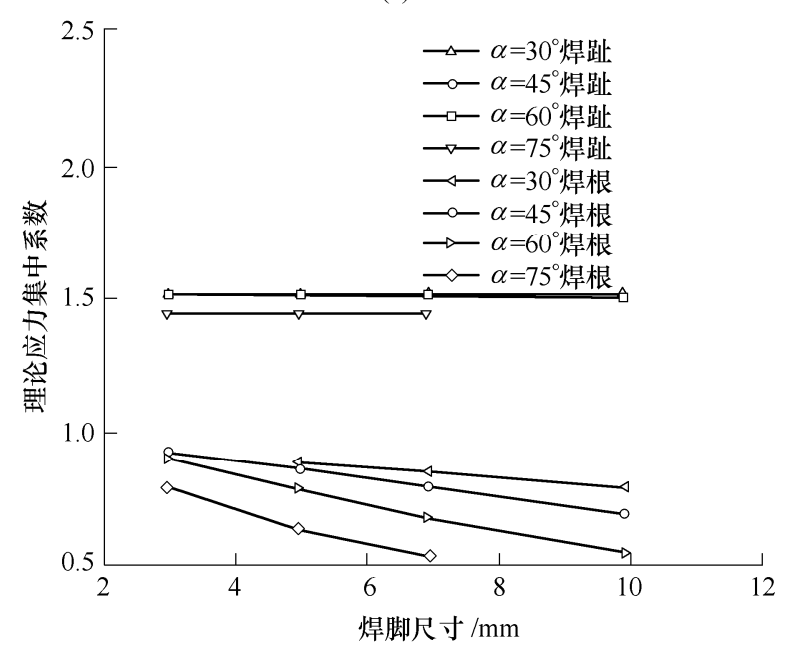

(b) $r=8 \mathrm{~mm}$

图 10 按最大主应力计算时焊脚尺寸的影响

由图 10 可知, 焊趾处的理论应力集中系数与 焊脚尺寸的关系不大, 仅当焊趾过度圆弧倒角半 径非常小时才随焊脚尺寸的增大而明显增大。焊 根处的理论应力集中系数则随焊脚尺寸的增加而 线性减小。

由于计算理论应力集中系数是线弹性分析, 计算结果与载荷的大小无关; 如果将焊脚尺寸 $K$ 、 焊趾过渡圆弧半径 $r$ 对板厚 $2 t$ 做归一化处理, 那 么计算结果也与板厚无关。将数据拟合得到所需 关系式。

按等效 Von Mises 应力计算, 焊趾理论应力集 中系数关系方程为

$$
K_{\mathrm{t}}^{\mathrm{toe}}=1+0.34\left(\frac{r}{2 t}\right)^{-0.65} \cos ^{0.35} \alpha
$$

按最大主应力计算, 焊趾理论应力集中系数关 系方程为

$$
K_{\mathrm{t}}^{\mathrm{toe}}=1+0.55\left(\frac{r}{2 t}\right)^{-0.53} \cos ^{0.27} \alpha
$$

按等效 Von Mises 应力计算, 焊根理论应力集
中系数关系方程为

$$
K_{\mathrm{t}}^{\mathrm{root}}=1.08-\left(\frac{K}{K+t}\right) \sin ^{2.46} \alpha
$$

按最大主应力计算，焊根理论应力集中系数关 系方程为

$$
K_{\mathrm{t}}^{\mathrm{root}}=1.13-\left(\frac{K}{K+t}\right) \sin ^{1.59} \alpha
$$

根据 $\mathrm{T}$ 型接头几何特点, 等承载设计求解过程 为: 将焊根理论应力集中系数关系方程和材料的性 能参数代入式(27)、(28), 求使(24)取极小值的焊趾 角度和焊脚尺寸; 将焊根理论应力集中系数关系方 程和材料的性能参数代入式(21), 结合上步求得的 焊趾角度和焊脚尺寸确定焊趾过渡圆弧半径; 验证 所求得的接头几何参数是否满足式(20), 若符合则 为等承载设计接头的焊趾过渡圆弧半径，若不符合 则联立式(20)、(21), 重新求取焊趾角度和焊趾过渡 圆弧半径。

取材料参数如表 1 所示，取坡口角度 $45^{\circ}$ ，离 缝 $2 \mathrm{~mm}$, 针边 $2 \mathrm{~mm}$, 板厚 $10 \mathrm{~mm}$, 设计等承载 $\mathrm{T}$ 型接头, 焊缝的几何参数: 焊脚尺寸 $K=6.5 \mathrm{~mm}$ 、焊 趾倾角 $\alpha=73^{\circ}$ 、焊趾过渡圆弧半径 $r=5 \mathrm{~mm}$ 。建立 弹塑性有限元模型进行计算。

\section{表 1 母材和焊缝金属的强度和塑性指标}

\begin{tabular}{ccccc}
\hline 位置 & $\begin{array}{c}\text { 屈服强度 } \\
\sigma_{\mathrm{s}} / \mathrm{MPa}\end{array}$ & $\begin{array}{c}\text { 屈服点应变 } \\
\varepsilon_{\mathrm{s}}\end{array}$ & $\begin{array}{c}\text { 抗拉强度 } \\
\sigma_{\mathrm{b}} / \mathrm{MPa}\end{array}$ & $\begin{array}{c}\text { 抗拉强度点应变 } \\
\varepsilon_{\mathrm{b}}\end{array}$ \\
\hline 母材 & 662 & 0.07 & 718 & 0.16 \\
焊缝 & 378 & 0.04 & 484 & 0.30 \\
\hline
\end{tabular}

图 11 14 是等承载设计 $\mathrm{T}$ 型接头不同位置的等 效 Von Mises 应力、最大主应力、等效塑性应变和 等效总应变的变化情况。

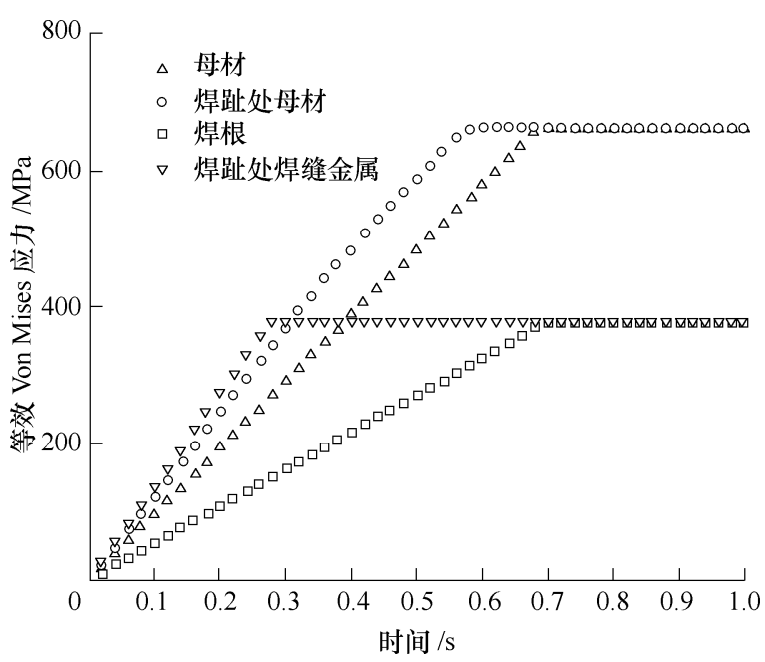

图 11 等效 Von Mises 应力的变化 


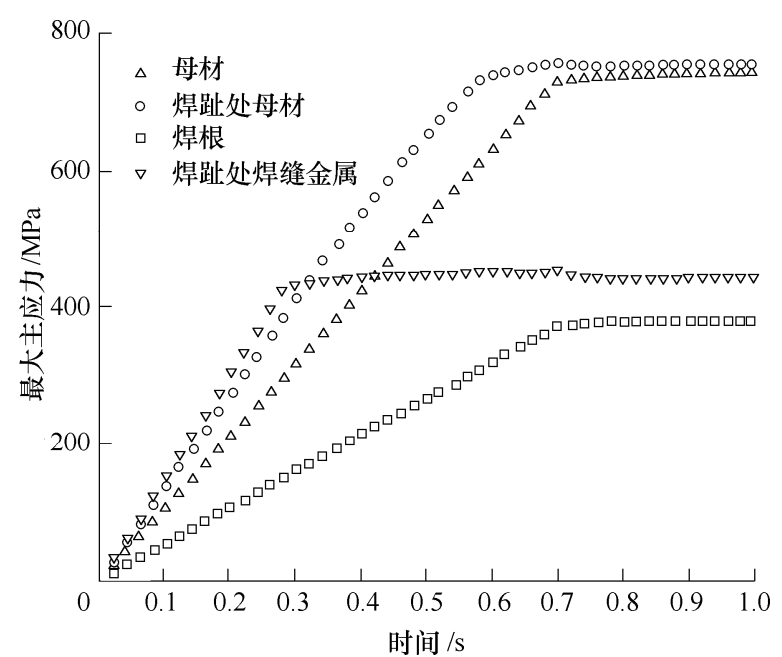

图 12 最大主应力的变化

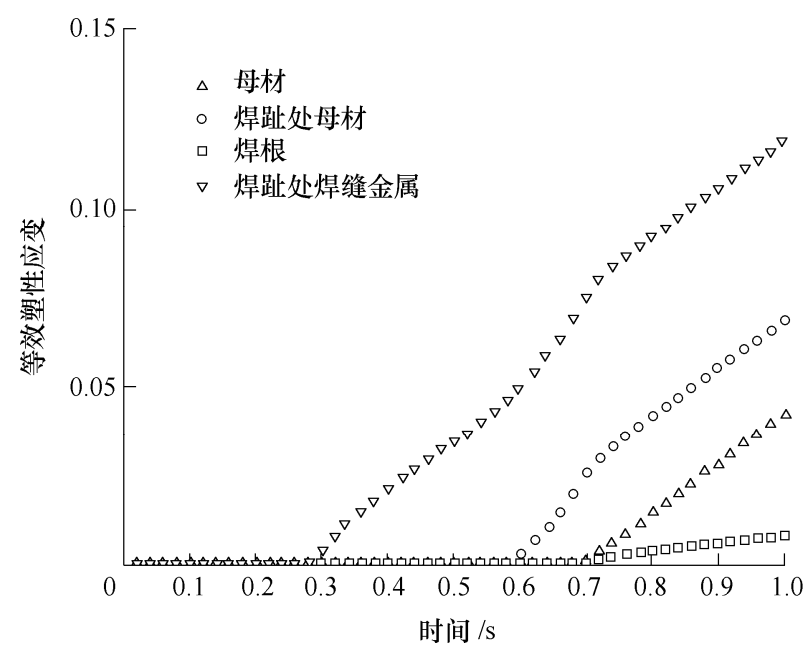

图 13 等效塑性应变的变化

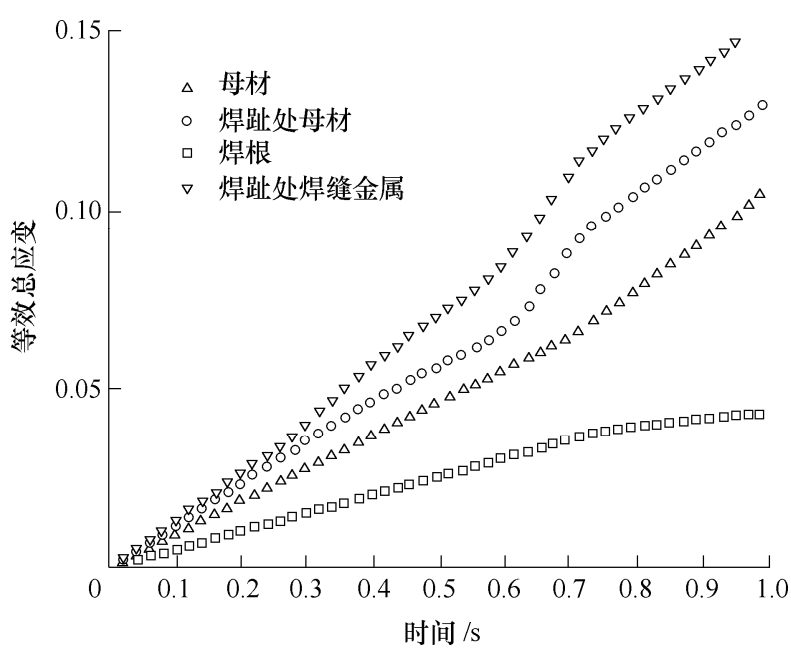

图 14 等效总应变的变化

可见，等承载设计 $\mathrm{T}$ 型接头变形比较均匀、协 调, 焊趾处的焊缝金属首先发生屈服, 但是其进一 步变形受到母材金属的限制, 随后焊趾处的母材发 生屈服, 在其塑性耗尽之前, 平滑母材与焊根处同 时发生屈服, 所以接头的承载能力达到了母材的屈 服水平。可以预见, 等承载设计 $\mathrm{T}$ 型接头的断裂模
式为全面屈服(焊缝)断裂, 断裂发生在焊趾处, 如 果进一步增大过渡圆弧半径或改进过渡方案, 消除 应力集中, 接头的断裂模式将变为全面屈服断裂甚 至全面屈服(母材)断裂, 承载能力达到母材的抗拉 强度。

\section{4 结论}

(1) 建立了低匹配焊接接头等承载基本理论, 通过定义广义匹配比和广义力学集中系数, 提出了 等承载的实现条件为广义力学集中系数等于广义匹 配比。该理论概括了等承载研究的规律, 可拓展等 承载设计应用范围。

(2) 针对接头等承载实现条件难以直接求解的 情形, 给出了焊接接头等承载设计的一般方法, 该 方法可简化设计，降低设计难度。

(3) 对承受静拉伸的 $\mathrm{T}$ 型接头进行了等承载设 计, 给出了具体的等承载实现条件和求解过程, 验 证了等承载基本理论和方法的普适性。

\section{参 考 文 献}

[1] 李亚江. 特殊及难焊材料的焊接 $[\mathrm{M}]$. 北京: 化学工业 出版社, 2003.

LI Yajiang.Welding of special and hard-to-weldmaterials [M]. Beijing: Chemical Industry Press, 2003.

[2] 拉达伊. 焊接结构疲劳强度 [M]. 北京: 机械工业出版 社, 1994.

RADAJ. Fatigue strength of welding structure $[\mathrm{M}]$. Beijing: China Machine Press, 1994.

[3] 武亚鹏, 侯建伟. 高强钢的焊接分析 [J]. 焊接与切割, 2004(4): 28-32.

WU Yapeng, HOU Jianwei. Welding analysis of high strength steel[J].Welding and Cutting, 2004(4): 28-32.

[4] 张炳范, 马元林, 曾悦坚, 等. 焊缝金属对高强钢热影 响区抗冷裂性的影响[J]. 焊接学报, 1991, 12(1): 31-38. ZHANG Bingfan, MA Yuanlin, ZENG Yuejian, et al. The effect of weld metal on the cold crack resistance of high strength steel[J]. Transactions of the China Welding Institution, 1991, 12(1): 31-38.

[5] 李亚江, 王娟, 刘鹏. 低合金钢焊接及其工程应用[M]. 北京: 化学工业出版社, 2003.

LI Yajiang, WANG Juan, LIU Peng. Low alloy steel welding and its engineering application[M]. Beijing: Chemical Industry Press, 2003.

[6] ALTINO J, LOUREIRO R. Effect of heat input on plastic deformation of undermatched welds[J]. Journal of 
Materials Processing Technology, 2002, 128(1): 240-249.

[7] 朱亮, 陈剑虹. 力学性能不均匀焊接接头的强度预测 [J]. 焊接学报, 2005, 26(5): 13-17.

ZHU Liang, CHEN Jianhong. The strength prediction of non-uniform welding joint $[J]$. Transactions of the China Welding Institution， 2005，26(5): 13-17.

[8] HEINZ D, RICHTER B, WEBER S. Application of advanced materials for ship construction-experiences and problems[J]. Materials and Corrosion, 2000, 51(6): 407-412.

[9] 杨建国, 黄鲁永, 张勇, 等. $30 \mathrm{CrMnSi}$ 钢 TIG 焊冷裂 纹形成机制 $[\mathrm{J}]$. 焊接学报, 2011，32(12): 13-16.

YANG Jianguo, HUANG Luyong, ZHANG Yong, et al. Mechanism of cold welding cracks in $30 \mathrm{CrMnSi}$ steel joints welded by TIG method[J]. Transactions of the China Welding Institution，2011，32(12): 13-16.

[10] TUMA J V, SEDMAK A. Analysis of the unstable fracture behaviour of a high strength low alloy steel weldment[J]. Engineering Fracture Mechanics, 2004, 71(9): 1435-1451.

[11] HAO S, SCHWALBE K H, CORNEC A. The effect of yield strength mismatch on the fracture analysis of welded joints: Slip-line field solutions for pure bending[J]. Int $\mathrm{J}$ Solids Struct, 2000, 37(39): 385-411.

[12] ROBERT R. Must preheat HY-80 welds[J]. IronAge, 1983, 226(13): 55-57.

[13] RODRIGUES D M, MENEZES L F, LOUREIRO A. Modelling the effect of HAZ undermatching on the crack-tip stress distribution in idealized welds[J]. International Journal of Mechanical Sciences, 2004, 46(10): 1481-1488.

[14] 徐智珍. 产品研制中焊接设计标准应用分析 [J]. 航空 标准化与质量, 2010(2): 46-48.

$\mathrm{XU}$ Zhizhen. Application analysis of welding design standard in product development[J]. Aeronautic Standardization and Quality, 2010(2): 46-48.

[15] 郭桃红. 焊接设计标准在燃气轮机研制中的应用 [J]. 航空标准化与质量, 2011(6): 13-15.

GUO Taohong. Application of welding design standard in gas turbine development[J].Aeronautic Standardization and Quality, 2011(6): 13-15.

[16] 杨建国, 周号, 雷靖, 等. 焊接应力与变形数值模拟领 域的若干关键问题 [J]. 焊接, 2014(3): 8-17.

YANG Jianguo, ZHOU Hao, LEI Jing, et al. Several key problems in numerical simulation of welding stress and deformation[J]. Welding and Joining, 2014(3): 8-17.
[17] 杨建国, 雷靖, 周号, 等. 关于焊接结构有限元分析的 思考 [J]. 焊接, 2014(12): 10-19.

YANG Jianguo, LEI Jing, ZHOU Hao, et al. Thoughtson finite element analysis of welded structures. Welding and Joining, 2014(12): 10-19.

[18] 赵智力. 基于等承载能力原则的高强钢低匹配焊接接 头设计[D]. 哈尔滨: 哈尔滨工业大学, 2009 .

ZHAO Zhili. Design of undermatching welded joints for high strength steels based on principle of the equal load-carrying capacity[D]. Harbin: Harbin Institute of Technology, 2009.

[19] 赵智力, 杨建国, 方洪渊, 等. 基于等承载能力原则的 低匹配对接接头设计 $[J]$. 焊接学报，2008，29(10): 93-96.

ZHAO Zhili, YANG Jianguo, FANG Hongyuan, et al. Design of undermatching butt joint based on equal load-carrying ability[J]. Transactions of the China Welding Institution, 2008, 29(10): 93-96.

[20] 赵智力, 方洪渊, 杨建国, 等. 低匹配对接接头的 “等 承载” 设计及拉伸疲劳行为 $[\mathrm{J}]$. 机械工程学报, 2010 , 46(10): 75-80.

ZHAO Zhili, FANG Hongyuan, YANG Jianguo, et al. Design of undermatchingbutt Joints for equal load-carrying capacity and tensile and fatigue behaviors of joints $[\mathrm{J}]$. Journal of Mechanical Engineering, 2010, 46(10): $75-80$.

[21] 程荣龙. 高强钢低匹配搭接接头的等承载设计 [D]. 哈 尔滨：哈尔滨工业大学, 2012.

CHEN Ronglong. Equal load-carrying design of under-matched ocerlapjoints[D]. Harbin: Harbin Institute of Technology, 2012.

[22] 王佳杰. 低匹配焊接接头弯曲等承载设计及随焊整形 [D]. 哈尔滨: 哈尔滨工业大学, 2015.

WANG Jiajie. Design and welding with trailing shape modifying of under-matched welded joint based on principle of the equal bending load-carrying capacity[D]. Harbin: Harbin Institute of Technology, 2015.

[23] 王佳杰，董志波，刘雪松，等.弹性阶段低匹配对接接 头三点弯曲余高形状设计 $[\mathrm{J}]$. 焊接学报, 2012, 33(8): $37-40$.

WANG Jiajie, DONG Zhibo, LIU Xuesong, et al. Shape design of reinforcement for under-matched butt joints under three-point bending load in elastic stage[J]. Transactions of the China Welding Institution, 2012, 33(8): $37-40$

[24] 王涛. 基于断裂参量 $\mathrm{K}$ 因子的焊接接头等承载设计 [D]. 
哈尔滨：哈尔滨工业大学, 2012.

WANG Tao. Equal load carrying capacity joint design based on fracture parameter of stress intensity factor[D].

Harbin: Harbin Institute of Technology, 2012.

[25] 雷靖. 基于断裂参量 $\mathrm{K}$ 的船用 945 钢对接接头抗冲击等 承载设计[D]. 杭州: 浙江工业大学, 2016.

LEI Jing. Thebearing impact equal load carrying capacity design of butt joint of ship-buliding 945 steel based on the fractureparameters K[D]. Hangzhou: Zhejiang University of Technology, 2016.

作者简介: 郭军礼, 男, 1987 年出生, 博士研究生。主要研究方向为焊 接结构力学及可靠性分析。

E-mail: iamgj1@163.com

董志波(通信作者), 男, 1975 年出生, 博士, 副教授, 博士研究生导师。 主要研究方向为焊接过程数值模拟与仿真技术、焊接结构可靠性及寿命 预测。

E-mail: dongzhb@hit.edu.cn

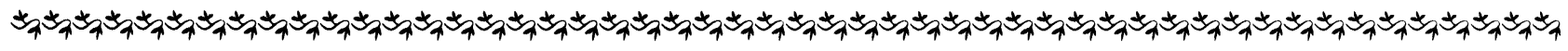

第 8 届上银优秀机械博士论文奖——优秀奖

\section{高精度激光差动共焦超长焦距测量方法与技术研究}

作 者: 李志刚

毕业学校: 北京理工大学

指导教师: 赵维谦

超长焦距透镜被广泛应用于激光聚变系统、空间光学系统和高能激光武器等大型光学系统研究领域中, 超长焦距测量精 度直接影响到大型光学系统的成像质量及使用性能。因此, 开展高精度超长焦距的测量方法和技术研究对于大型光学系统具 有重要应用价值。

论文主要完成了以下工作:

开展了一种具有参考镜焦距和组合透镜间隔自校准能力的激光差动共焦组合焦距测量方法研究, 利用差动共焦精密定焦 技术，对参考镜焦点及其后表面顶点精确定位测得参考镜焦距值、对被测镜后表面顶点和参考镜后表面顶点精确定位测得轴 向间隔、对有/无被测镜时系统焦点精确定焦测得焦点位置变化量, 由组合焦距计算公式间接测得被测镜焦距。

构建了激光差动共焦组合超长焦距测量光路, 建立了激光差动共焦组合超长焦距测量光路理论分析模型, 为测量光路中 关键影响因素分析和测量系统研制提供理论依据。

提出了参考镜焦距和镜组间隔自校准方法, 该方法基于系统焦点与差动共焦光强响应曲线零点精确对应这一特性, 对参 考镜焦点及其后表面顶点进行精确定位实现参考镜焦距自校准, 对参考镜后表面顶点及被测镜后表面顶点进行精确定位实现 镜组间隔自校准，解决了组合测量法中参考镜焦距和镜组间隔校准难题。

提出了一种激光差动共焦超大曲率半径测量方法, 该方法利用光束会聚点与差动共焦光强响应曲线零点精确对应这一特 性, 对被测镜猫眼位置和参考镜后表面顶点位置进行精确定位, 获得被测镜移动距离, 利用光线追迹算法, 实现曲率半径高 精度测量。

参与研制了首台测量口径 $610 \mathrm{~mm}$ 超长焦距测量系统, 分析了主要误差源及影响机理, 开展了超长焦距试验研究, 采用 蒙特卡洛法进行不确定度评定。试验结果表明, 对 $31.2 \mathrm{~m}$ 焦距相对重复测量精度为 $0.0034 \%(34 \mathrm{ppm})$, 对 $12 \mathrm{~m}$ 焦距相对重 复测量精度为 $0.0044 \%(44 \mathrm{ppm})$ 。

综上所述, 本论文提出了一种具有参考镜焦距和组合透镜间隔自校准能力的激光差动共焦超长焦距测量方法, 基于所提 方法研制了一套大口径超长焦距测量系统, 验证了所提方法可行性, 为解决大口径超长焦距的高精度测量难题提供了一条全 新的技术途径。 